\title{
Synthesis of Angle Arrays by the NDF of the Radiation Integral
}

\author{
Giovanni Leone, Fortuna Munno, and Rocco Pierri
}

\begin{abstract}
Linear arrays have been well studied for their widespread applications. However, they suffer from severe limitations in their scanning capabilities due to the inherent main beam spreading. The combination of two linear arrays, radiating simultaneously, may provide the coverage of a wide angular domain by identical beams. More in general, first, we consider the radiation properties of a collection of continuous linear sources by following an inverse source approach which aims at investigating the spectral decomposition of the relevant radiation operator by discussing its Singular Value Decomposition and the Number of Degrees of Freedom of the source according to its geometry. Then a connection with the known closed form results of the single line case is established. Next, for an angle geometry, the discussion of the Point Spread Function reveals the condition to be achieved for an angle source to radiate identical beams with an angular coverage larger than the one of a single linear source with the same total length. Finally, the approach is applied to linear arrays, arranged in an angle geometry, also including the effect of the element factor. Numerical results about the synthesis of identical focused radiation patterns demonstrate the usefulness of the approach.
\end{abstract}

Index Terms - Conformal antennas, Electromagnetic scattering inverse problems, Inverse source problem, Multifaceted arrays, Number of Degrees of Freedom, Singular Value Decomposition.

\section{INTRODUCTION}

C ONFORMAL, i.e. non planar, antennas are attracting wide interest because of their geometrical and electrical properties. In fact, they exhibit aero dynamic features useful for sensing and communication purposes on vessels, aircrafts and vehicles. In addition, the greater latitude provided by the additional spatial dimension can enlarge the class of patterns that can be radiated, and thus provide new electrical features.

For instance, the scanning capabilities of a linear array are limited as the beam maximum approaches the broadside directions, where the radiation pattern naturally degrades as it enlarges. Therefore, a further dimension to the source is added by devising multiple arrays' solutions to form the so-called multi-faceted radar antennas [1,2], so as to achieve a full angle coverage with similar beams. However, the arrays are usually fed one at a time, and the electrical advantages of the conformal

Paper submitted on January 2020.

"This work was supported in part by the Italian Ministry of University and Scientific Research under PRIN 2017 Programme." geometry are not fully exploited. In [3] a simultaneous operation of the array facets is considered but only from the scanning time point of view without taking into account the complete radiation pattern of the antenna.

In addition, the antenna system design for the optimal geometrical configuration of the additional arrays is mostly performed under arguments accounting for the maximum acceptable scanning angle in dependence of the scan angles, scan losses, antenna dimensions, geometric directivity, and total number of antenna elements [4]. The electrical performances of the array concerning the antenna gain, accounting for the beam broadening and element impedance variations due to mutual coupling, are taken into account in [5] according to a heuristic approach leading to the introduction of an equivalent geometrical parameter. Again, the simple above mentioned analyses do not take into account the radiation properties of the entire antenna.

Generally speaking, the radiation behavior of a source also depends on its geometry, and a full investigation requires the adoption of an inverse source problem approach and the analysis of the mathematical properties of the pertinent radiation operator. This concerns the reconstruction of a current function from the knowledge of the far field, that is the inversion of the integral relationship which connects them.

However, the ill-conditioning behavior [6] of the relevant integral operator implies that only a finite number of source current functions can be correctly reconstructed in presence of uncertainties on data, otherwise a small noise on data can provide a large error on the reconstructed current. Therefore, the Number of Degrees of Freedom (NDF) $[7,8,9,10]$ of the source current in connection to its geometry should be introduced as the (finite) number (i.e. the dimension of the subspace) of the source current functions that can be stably reconstructed [11]. This approach was applied in [12,13] to establish the NDF of a curve source analytically, both in closed form and as upper bounds.

Besides, as well known, the physically realizable radiation patterns are connected to the source geometry and require the knowledge of the NDF, which, in turn, define the number (i.e., the dimension of the subspace) of the radiation pattern functions that can be radiated by a finite energy source. Then, it is possible to compare the radiation properties of the different

G. Leone, F. Munno and R. Pierri are with the Dipartimento di Ingegneria, Università della Campania "L. Vanvitelli", Aversa, Italy (e-mail: giovanni.leone@unicampania.it, fortuna.munno@unicampania.it, rocco.pierri@unicampania.it). 
sources by their capabilities of radiating a far field focusing (ideally) on one direction. Therefore, the investigation of the behavior of the so-called Point Spread Function (PSF) [14,15] in the far field set can provide the answer to this problem.

In this paper we discuss the features of the inverse source problem for some geometries composed of a combination of arbitrary linear sources, starting from the closed form results available for a single linear source. This approach may reveal itself useful in order to establish a way to compare their general radiation properties before undertaking the synthesis procedure of a particular antenna with defined specifications [16].

A useful tool to perform the above mentioned tasks consists in the spectral decomposition of the relevant radiation operator by the Singular Values Decomposition (SVD) [17].

In Section II, we formulate the inverse problem and we recall and employ the known results concerning the SVD of a single linear source for the geometry at hand in order to provide an estimate of the NDF, extending the results of [18] to a generic case. Section III is devoted to the verification of the provided estimate against exact, numerically computed results for different source geometries. We introduce the PSF in Section IV, together with an analytical approximation of it. Then, with reference to an angle geometry with identical sides, we establish a comparison between different geometries by means of the PSF behavior. We present an application of the previous theoretical results to the synthesis of a continuous source capable of radiating identical beams pointing at different directions in Section V. Then, in Section VI, we extend the analysis and the results to array antennas, arranged at an angle geometry, including a synthesis example, as well. Thus, the two new features of the approach, accounting for the full radiation behavior of the source and the simultaneous operation of more than one array, can be applied to faceted arrays. Conclusions follow in Section VII.

\section{LINEAR SOURCES}

Since our goal is to examine the radiation properties of a source consisting of a generic number $M$ of arbitrarily oriented linear sources, we start by specifying the reference configuration and the relevant radiated field. By assuming invariance along the $y$ axis, the addressed problem becomes scalar and two-dimensional. The $n^{\text {th }}$ linear source is $2 b_{n}$ long, it has a center point with rectangular coordinates $\left(x_{0 n}, z_{0 n}\right)$ and forms an angle $\phi_{0 n}$ with the $z$ axis (Fig. 1). Moreover, it hosts a (surface) current density $J_{n}(\cdot, \cdot)$, producing a radiated field

$E_{n}(\theta)=\int_{-b_{n}}^{b_{n}} J_{n}\left(x_{n}(s), z_{n}(s)\right) e^{j \beta\left[x_{n}(s) \sin \theta+z_{n}(s) \cos \theta\right]} d s$,

over the whole $360^{\circ}$ angle spanned by $\theta$, with $\beta=2 \pi / \lambda$ being the wave number, $x_{n}(s)=x_{0 n}-s \sin \phi_{0 n}, z_{n}(s)=$ $z_{0 n}-s \cos \phi_{0 n}$, and $s$ representing the arc length variable.

Equation (1) allow us to define a radiation operator $\mathcal{T}_{n}$ for each line as

$\mathcal{T}_{n}: J_{n} \in L_{\left[-b_{n}, b_{n}\right]}^{2} \rightarrow E_{n} \in L_{[-\pi, \pi]}^{2}$, where $L_{[,,]}^{2}$ is the set of all the finite energy functions supported in the interval of the subscript. As a consequence of the linearity of the equations, the total field is given by the superposition of the fields in (1)

$E(\theta)=\sum_{n=1}^{M} E_{n}(\theta)$.

Accordingly, to each set of $M$ square integrable current densities we can associate a radiated field by means of the operator $\mathcal{T}$ written as

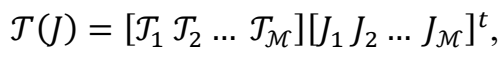

where $t$ denotes the transposition.

A useful tool to examine the properties of operator (4) is represented by its SVD. It consists of the singular values $\left\{\sigma_{n}\right\}$ and the singular functions $\left\{u_{n}(\theta), v_{n}(\underline{r})\right\}$, if $\underline{r}=$ $\left(x_{n}(s), z_{n}(s)\right)$ denotes a source point, corresponding to the eigenvalues and eigenfunctions solving the shifted eigenvalue problem $\mathcal{L}\left(v_{n}\right)=\sigma_{n} u_{n}$ and $\mathcal{L}^{\dagger}\left(u_{n}\right)=\sigma_{n} v_{n}$, where the superscript $\dagger$ indicates the adjoint operator.

When the source geometry is a single $2 b$ long line and the observation domain spans the interval $\theta \epsilon\left(0^{\circ}, 180^{\circ}\right)$, by introducing the spectral variable $w=\cos \theta$ the relevant operator to be considered can be recast as

$E(w)=\int_{-b}^{b} J(x) e^{j \beta x w} d x$

where $w \epsilon(-1,1)$. The SVD of (3) is known [19] in terms of the so-called prolate spheroidal functions. An important result of [20] concerns the behavior of the singular values $\sigma_{n}$, that are nearly constant for $n<N_{s}=2[\beta b / \pi]$, before the typical exponential decay. When the observation variable $\theta$ spans the whole $2 \pi$ angular range, although the singular values of (5) are not the same as those of the operator $\mathcal{J}_{1}$, the NDF is the same for both the cases and the only difference is in the shaping of their behavior [21].

The exact evaluation of the NDF as

$$
N_{S} \approx\left[\frac{2 \beta b}{\pi}\right],
$$

still holds when the single line source is translated and rotated, as in our case. However, no analytical evaluation nor estimation is available when the linear sources are more than one and, at first sight, only numerical results can be obtained. However, analytical argumentations can be proposed in order to deduce an estimation of the NDF, even when the lines are combined to form a polygonal shape.

Let us start with the simpler case of two linear sources with comparable dimensions before proceeding with a more complex and general configuration. The relevant operator (4) to be considered can be written as

$\mathcal{T}(J)=\left[\begin{array}{ll}\mathcal{T}_{1} & \mathcal{T}_{2}\end{array}\right]\left[\begin{array}{l}J_{1} \\ J_{2}\end{array}\right]$. 
For the purpose of NDF estimation, we can, equivalently, consider the operator

$\left(\mathcal{T}^{\dagger} \mathcal{T}\right)(J)=\left[\begin{array}{ll}\mathcal{T}_{1}^{\dagger} \mathcal{T}_{1} & \mathcal{T}_{1}^{\dagger} \mathcal{T}_{2} \\ \mathcal{T}_{2}^{\dagger} \mathcal{T}_{1} & \mathcal{T}_{2}^{\dagger} \mathcal{T}_{2}\end{array}\right]\left[\begin{array}{l}J_{1} \\ J_{2}\end{array}\right]$

obtained by resorting to the adjoint $\mathcal{T}^{\dagger}$ of the operator $\mathcal{T}$, since the number of significant eigenvalues of (8) will be equal to the number of relevant singular values of (7). To this end, we recall that, by definition, $\mathcal{T}^{\dagger}$ can be expressed as

$\mathcal{T}^{\dagger}=\left[\begin{array}{llll}\mathcal{T}_{1}^{\dagger} & \mathcal{T}_{2}^{\dagger} & \ldots & \mathcal{T}_{M}^{\dagger}\end{array}\right]^{t}$

with

$\mathcal{T}_{n}^{\dagger}(E)=\int_{-\pi}^{\pi} E(\theta) e^{-j \beta\left[x_{n}(s) \sin \theta+z_{n}(s) \cos \theta\right]} d \theta$.

If we introduce the indices $i, j \in\{1,2\}$ to specify which source we are referring to, each operator in (8) can be written explicitly as

$\left(\mathcal{T}_{i}^{\dagger} \mathcal{T}_{j}\right)\left(J_{j}\left(s^{\prime}\right)\right)=\int_{-b_{j}}^{b_{j}} J_{j}(s) K_{i, j}\left(s, s^{\prime}\right) d s$,

where $K_{i, j}\left(\mathrm{~s}, \mathrm{~s}^{\prime}\right)$ is the kernel of the integral operator $\mathcal{T}_{i}^{\dagger} \mathcal{J}_{j}$, and is provided by the Bessel function $J_{0}(\cdot)$ of first kind and zero-th order, as,

$K_{i j}\left(s, s^{\prime}\right)=\int_{-\pi}^{\pi} e^{j \beta\left[\left(x_{j}(s)-x_{i}\left(s^{\prime}\right)\right) \sin \theta+\left(z_{j}(s)-z_{i}\left(s^{\prime}\right)\right) \cos \theta\right]} d \theta=$

$2 \pi\left\{\begin{array}{c}J_{o}\left(\beta\left|s-s^{\prime}\right|\right), i=j \\ J_{o}\left(\beta \sqrt{\left(x_{j}(s)-x_{i}\left(s^{\prime}\right)\right)^{2}+\left(z_{j}(s)-z_{i}\left(s^{\prime}\right)\right)^{2}}\right), i \neq j\end{array}\right.$

The four terms in (8) might be compared and "weighted" according to their respective operator norm. Unfortunately, a closed form evaluation of them is not easy to be achieved.

However, since an upper bound can be provided by the kernel function norm, as $\left\|\mathcal{T}_{i}^{\dagger} \mathcal{T}_{j} J_{j}\right\| \leq\left\|K_{i, j}\right\|\left\|J_{j}\right\|$ [22], we can resort to it to provide an indication of how each operator term impacts on the resulting operator (8). In particular, we find that

$$
\left\{\begin{array}{c}
\left\|K_{i, j}\left(s, s^{\prime}\right)\right\|=(2 \pi)^{2} \\
\left(\iint_{-b_{i}}^{b_{i}}\left|J_{0}\left(\beta\left|s-s^{\prime}\right|\right)\right|^{2} d s d s^{\prime}\right)^{\frac{1}{2}} \\
\left(\int_{-b_{i}}^{b_{i}} \int_{-b_{j}}^{b_{j}}\left|J_{0}\left(\beta \sqrt{\left(x_{j}(s)-x_{i}\left(s^{\prime}\right)\right)^{2}+\left(z_{j}(s)-z_{i}\left(s^{\prime}\right)\right)^{2}}\right)\right|^{2} d s d s^{\prime}\right)^{\frac{1}{2}} \quad i=j
\end{array}\right.
$$

In both of the integrals, the same Bessel function is observed for different arguments. Since the Bessel function is maximum when its argument is close to zero, we can notice that, if $i=j$, for any $s \in\left[-b_{i}, b_{i}\right]$ there exists a point $s^{\prime}$ in the same interval such that the argument is zero. On the contrary, if $i \neq j$ the Bessel function argument will be zero only in the intersection point between the two linear sources, if it exists, and the relevant norm term will be negligible for sources with comparable lengths. Moreover, when the distance between the lines is grater, the Bessel function argument can be expected to be larger, so that its contribution to the relevant norm term will decrease by increasing the angle between the two lines or the minimum distance between them. This means that the kernel norm and, accordingly, also the operator norm, is expected larger for the diagonal contributions than for the off-diagonal ones. Therefore, it results,

$\left(\mathcal{T}^{\dagger} \mathcal{T}\right)(J) \approx\left[\begin{array}{cc}\mathcal{T}_{1}^{\dagger} \mathcal{T}_{1} & 0 \\ 0 & \mathcal{T}_{2}^{\dagger} \mathcal{T}_{2}\end{array}\right]\left[\begin{array}{l}J_{1} \\ J_{2}\end{array}\right]$

Since the eigenvalues of a block diagonal operator are the combination of the ones of each individual term, by denoting with $N_{s 1}$ the NDF of the source denoted by subscript 1 and with $N_{s 2}$ the NDF of the source 2, respectively, we find that the NDF of the whole configuration can be provided by

$N \approx N_{s 1}+N_{s 2}$

This result indicates that the functional space of the total current can be approximately decomposed under two individual orthogonal subspaces.

By extending the previous discussion to the case of an arbitrary number $M$ of sources, (14) becomes

$\left(\mathcal{T}^{\dagger} \mathcal{T}\right)(J) \approx\left[\begin{array}{cccc}\mathcal{J}_{1}^{\dagger} \mathcal{T}_{1} & & & \\ & \mathcal{T}_{2}^{\dagger} \mathcal{T}_{2} & & \\ 0 & & \ddots & \\ 0 & & & \mathcal{T}_{M}^{\dagger} \mathcal{T}_{M}\end{array}\right]\left[\begin{array}{c}J_{1} \\ J_{2} \\ \vdots \\ J_{M}\end{array}\right]$

and we obtain a result analogous to (15), which is

$N \approx \sum_{n=1}^{M} N_{s n}$

where $N$ stands for the NDF of the whole set of line sources and $N_{s n}$ is the NDF of each individual linear source.

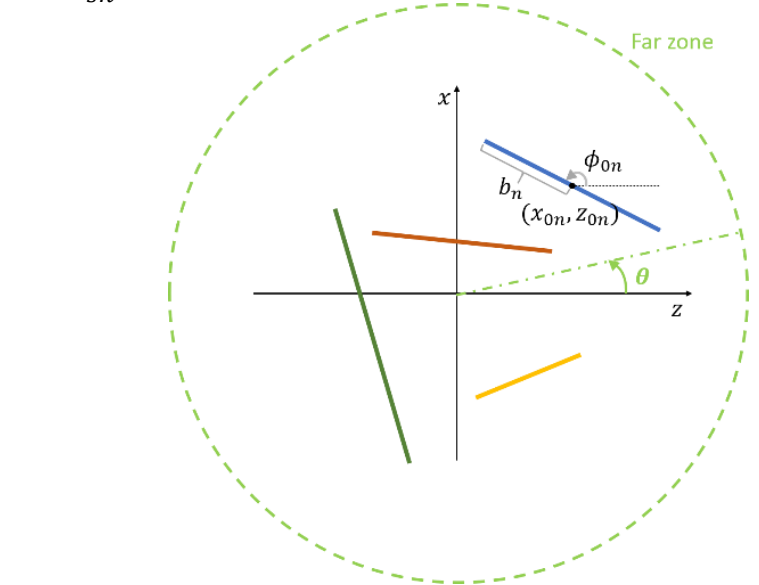

Fig. 1 A set of $M(4)$ rotated and translated linear sources.

\section{RESULTS CONCERNING THE NDF}

In this Section, first we are going to confirm with an example the results of Section II about the NDF evaluation. To this end we considered firstly an angle source consisting in two linear 
sources connected at one vertex, because this geometrical configuration is very useful in the analysis of the combination of linear arrays. Then we choose to consider a polygonal source mimicking a circumference source for which analytical results were available.

\section{A. Angle source}

We refer to the angle source consisting in two linear sources as in fig. 2 shaping an angle geometry, with $\phi_{02}=360^{\circ}-\phi_{01}$, $x_{02}=-x_{01}$ and $z_{01}=z_{02}$ and, hence, the same length $b_{2}=$ $b_{1}=b$ (Fig. 2).

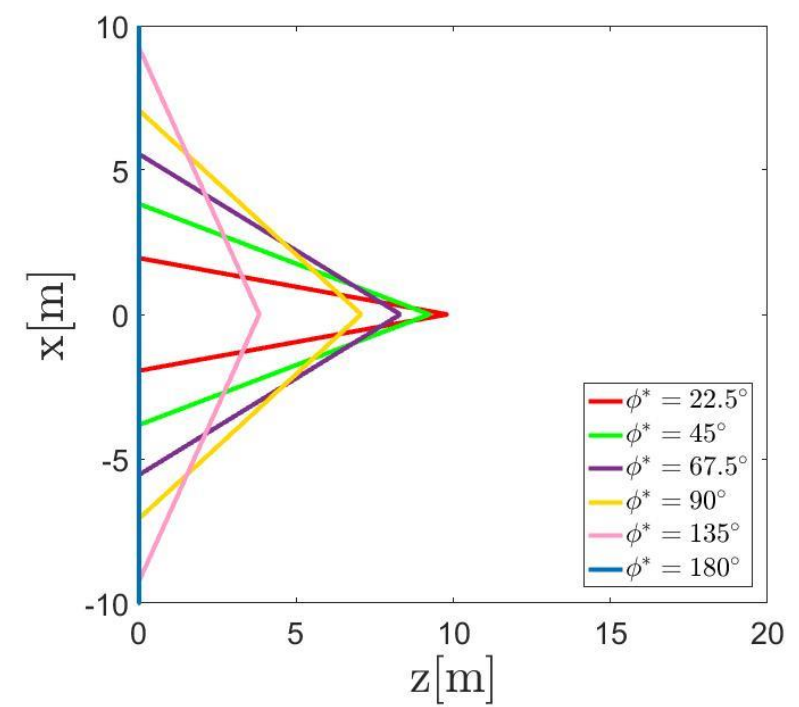

Fig. 2 Angle geometries increasing the angle $\phi^{*}=2\left(180^{\circ}-\phi_{01}\right)$ between the two linear sources. Each linear source has a semi-length $b_{1}=b_{2}=5 \lambda$, so that the whole length for each angle geometry is $20 \lambda$.

The first analysis concerns the NDF of the angle source. Because of the results of Section II, we compute (13) (Table 1) for different angle apertures $\phi^{*}=2\left(180^{\circ}-\phi_{01}\right)$ in order to appreciate the role of the second line. It can be observed that, by increasing the angle between the sources, the contributions of the mixed operators in (10) are reduced (see Table 1, where, for the chosen example $\left\|K_{1,1}\right\| /\left\|K_{1,2}\right\|=\left\|K_{2,2}\right\| /\left\|K_{2,1}\right\|$ ). Therefore, it can be expected that the number of significant singular values gets close to that one of a $4 b$ long linear source.

TABLE I.

\begin{tabular}{|c|c|}
\hline THE RATIO B & ||$K_{1,1}||$ AND ||$K_{1,2} \mid$ \\
\hline$\phi^{*}$ & ||$K_{1,1}|| /|| K_{1,2}||$ \\
\hline $22.5^{\circ}$ & 1.7880 \\
\hline $45^{\circ}$ & 2.1243 \\
\hline $67.5^{\circ}$ & 2.3710 \\
\hline $90^{\circ}$ & 2.5656 \\
\hline $135^{\circ}$ & 2.8112 \\
\hline $180^{\circ}$ & 2.8920 \\
\hline
\end{tabular}

This expectation is confirmed by the numerical computation of the singular values of (7) (Fig. 3).

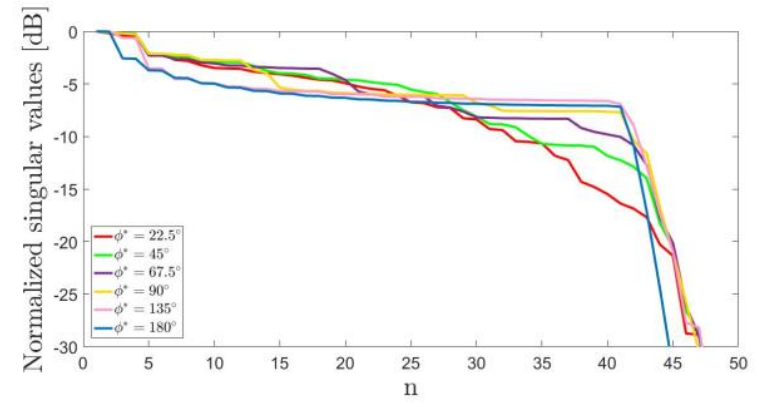

Fig. 3 Normalized singular values of the angle geometries in Fig. 2.

Therefore, by increasing $\phi^{*}$, the angle configuration gets closer to a linear source, and so does the singular values behavior does. It can be concluded that, for $\phi^{*} \geq 45^{\circ}$, the NDF can be always approximated by (15).

It is worth pointing out that this result does not imply that the far field subspaces spanned by the different source geometries are identical, but only that they approximately have the same dimension. Therefore, it is worth investigating those subspaces, finding a way to perform a rough comparison between them and examining the general behavior of the different radiation patterns they may radiate. The analysis of Section III introduces a way to achieve this goal.

\section{B. Polygonal source}

Now, we turn to a closed polygonal source. Then, because of (17), if we denote by $p$ the perimeter of the source, we can obtain an estimate of its NDF, by expressing (6) in terms of $\lambda$, as

$N \approx 2\left(\left[\frac{2 b_{1}}{\lambda}\right]+\ldots+\left[\frac{2 b_{M}}{\lambda}\right]\right)=2\left[\frac{p}{\lambda}\right]$.

As an example, let us consider an irregular quadrilateral, (see Fig. 4). For this geometry, $p=95.3 \lambda$ and (18) returns an NDF estimation of $N \approx 190$. Fig. 5 reports the numerically computed behavior of the singular values of (4): it is apparent that $\mathrm{N}$ is also the index from which the singular values behavior changes abruptly its slope and the singular values start to decay exponentially.

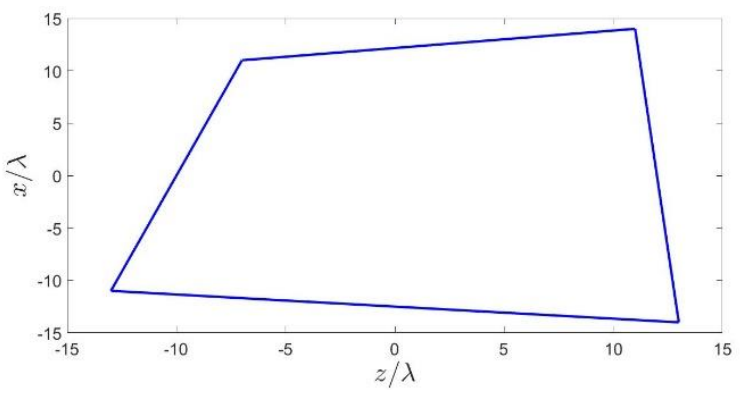

Fig. 4 Generic quadrilateral source. 


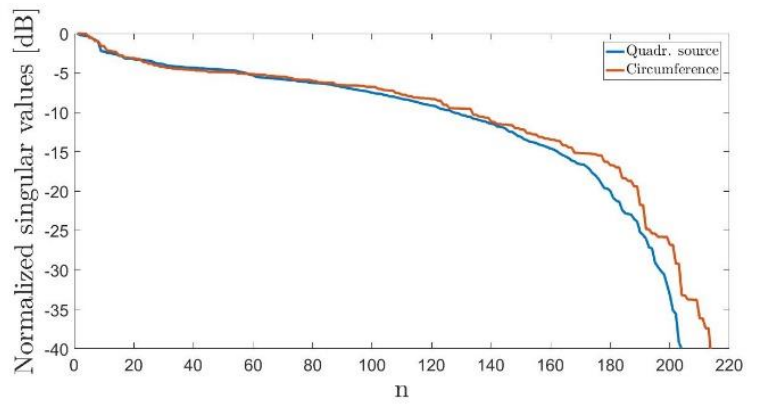

Fig. 5 Normalized singular values of operator (4) for the source of Fig. 4 (blue line) and for a circumference source with the same length (red line).

This result is consistent with the one of a circumference source of radius $a$. In fact, the SVD of the relevant radiation operator is analytically known [12] and the pertinent NDF is provided by the singular values' analytical expression, as,

$N_{c} \approx 2[\beta a]+1$.

which can be expressed explicitly in terms of its perimeter $p$, by writing $\beta$ in terms of wavelength $\lambda$, as

$N_{c} \approx 2\left[\frac{p}{\lambda}\right]$.

\section{POINT SPREAD FUNCTION}

By its very definition, the NDF provides the dimension of the subset of source current functions that can be reconstructed stably by a linear inversion algorithm. At the same time, it provides the dimension of the corresponding set of far fields that can be represented as a linear combination of the $u_{n}(\theta)$ basis functions. Since each source geometry may radiate a different class of patterns, it is difficult to provide a simple way to compare their performances on the whole.

However, in many circumstances, it is worth examining the capabilities of the source geometry to radiate an ideal focusing beam pointing at different directions $\theta_{0}$. From a mathematical point of view, this amounts to investigating the Point Spread Function (PSF) in the observation domain, defined as the impulsive response of the system made up by the cascade of the regularized inverse and the direct operator, which is given by

$\operatorname{PSF}\left(\theta, \theta_{0}\right)=\left(\mathcal{J T}^{-1}\right) \delta\left(\theta-\theta_{0}\right)$.

When a truncated SVD (TSVD) inversion scheme is adopted to perform the regularized inversion step, the $P S F$ writes explicitly as

$\operatorname{PSF}\left(\theta, \theta_{0}\right)=\sum_{n=1}^{N D F} u_{n}(\theta) u_{n}^{*}\left(\theta_{0}\right)$

and, when we neglect all the side lobes, the width of its main lobe describes how much the focusing field radiated by the considered source geometry spreads around its maximum.

Unfortunately, the $u_{n}^{\prime} \mathrm{s}$ left singular functions of operator (4) in (22) are not known in closed form for a general source composed of linear elements and a different approach must to be exploited, so as to avoid tackling a numerical problem for each new case.

Following the discussion in $[14,15]$ a good approximation of the PSF can be obtained when the inverse operator in (21) may be approximated by the adjoint one. This assumption requires that the singular values of the radiation operator should exhibit a step-like behavior, but it may assure good performances also when this is not the case provided the singular values exhibit an exponential decay. Therefore, to be used in the following, we define

$\widetilde{P S F}\left(\theta, \theta_{0}\right)=\left(\mathcal{T J}^{\dagger}\right) \delta\left(\theta-\theta_{0}\right)$

which reads as

$$
\begin{gathered}
\left(\mathcal{T J}^{\dagger}\right) \delta\left(\theta-\theta_{0}\right)= \\
{\left[\mathcal{T}_{1} \mathcal{T}_{1}^{\dagger}+\mathcal{T}_{2} \mathcal{T}_{2}^{\dagger}+\ldots+\mathcal{T}_{M} \mathcal{T}_{M}^{\dagger}\right] \delta\left(\theta-\theta_{0}\right)}
\end{gathered}
$$

In addition, for the geometries under consideration, an analytical approximation of (23) can be derived resorting to the adjoint operator (10) since

$$
\begin{gathered}
\left(\mathcal{T}_{n} \mathcal{T}_{n}^{\dagger}\right) \delta\left(\theta-\theta_{0}\right)= \\
2 b_{n} e^{j \beta\left[x_{0 n}\left(\sin \theta-\sin \theta_{0}\right)+z_{0 n}\left(\cos \theta-\cos \theta_{0}\right)\right]} \\
\operatorname{sinc}\left\{\beta b_{n}\left[\cos \left(\theta_{0}-\phi_{0 n}\right)-\cos \left(\theta-\phi_{0 n}\right)\right]\right\}
\end{gathered}
$$

Accordingly, (25) returns this analytic expression

$$
\begin{gathered}
\widetilde{P S F}\left(\theta, \theta_{0}\right)= \\
2 \sum_{n=1}^{M} b_{n} e^{j \beta\left[x_{0 n}\left(\sin \theta-\sin \theta_{0}\right)+z_{0 n}\left(\cos \theta-\cos \theta_{0}\right)\right]} . \\
\operatorname{sinc}\left\{\beta b_{n}\left[\cos \left(\theta_{0}-\phi_{0 n}\right)-\cos \left(\theta-\phi_{0 n}\right)\right]\right\}
\end{gathered}
$$

Fig. 6 compares the results of (22) and (23) in a typical test for an angle source when $b_{1}=b_{2}=b, x_{02}=-x_{01}$ and $\phi_{02}=$ $-\phi_{01}$, so that (26) becomes

$$
\begin{gathered}
\widetilde{P S F}\left(\theta, \theta_{0}\right)=2 b e^{j \beta z_{01}\left(\cos \theta-\cos \theta_{0}\right)}\left\{e^{j \beta x_{01}\left(\sin \theta-\sin \theta_{0}\right)}\right. \\
\operatorname{sinc}\left\{\beta b\left[\cos \left(\theta_{0}-\phi_{01}\right)-\cos \left(\theta-\phi_{01}\right)\right]\right\}+ \\
+e^{-j \beta x_{01}\left(\sin \theta-\sin \theta_{0}\right)} \\
\left.\operatorname{sinc}\left\{\beta b\left[\cos \left(\theta_{0}+\phi_{01}\right)-\cos \left(\theta+\phi_{01}\right)\right]\right\}\right\}
\end{gathered}
$$

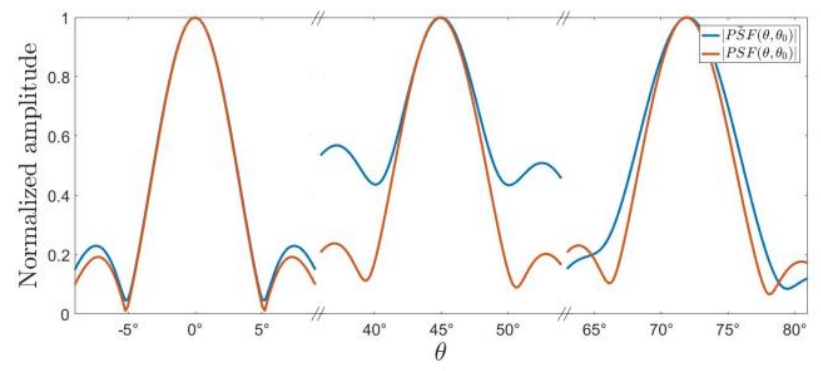

Fig. 6 Comparison of normalized actual (red line) and approximate (blue line) PSFs for an angle source with $\phi^{*}=67.5^{\circ}, b=5 \lambda$ for $\theta_{0}=0^{\circ}, 45^{\circ}, 72^{\circ}$.

By direct inspection it results $\widetilde{P S F}\left(-\theta,-\theta_{0}\right)=\widetilde{P S F}\left(\theta, \theta_{0}\right)$, $\widetilde{P S F^{*}}\left(180^{\circ}-\theta, 180^{\circ}-\theta_{0}\right)=\widetilde{P S F}\left(\theta, \theta_{0}\right)$ and $\widetilde{P S F^{*}}(\theta-$ $\left.180^{\circ},-\theta_{0}-180^{\circ}\right)=\widetilde{P S F}\left(\theta, \theta_{0}\right)$. This means that the modulus of $\widetilde{P S F}$ is an even function of $\theta$ and that the knowledge 
of the $\widetilde{P S F}$ in a quadrant is sufficient to derive it for the corresponding supplemental angles. A graphical summary of these symmetry results is summarized under Fig. 7. Therefore, we can exploit these considerations to restrict the following analysis just to the angular interval $\left[0,90^{\circ}\right]$.

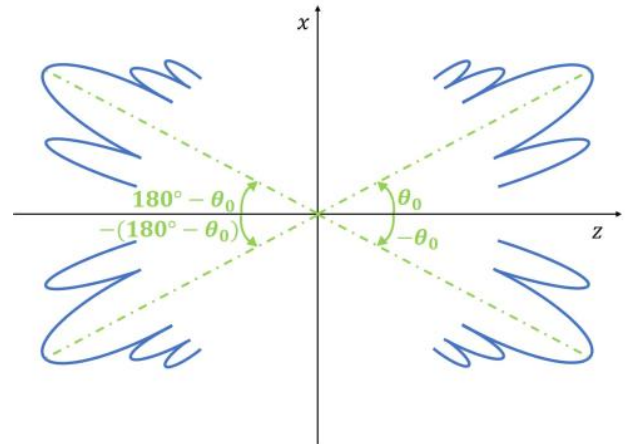

Fig. 7 Summering picture of the PSFs' amplitude symmetry properties when an angle source is considered.

Fig. 6 reveals that the approximated PSF main lobe follows exactly the actual one within a $2 \Delta \theta_{t h}$ wide angular domain around the maximum direction for different $\theta_{0}$. Therefore, if we are not interested in the side lobes directions, the radiation capability of the source can be appreciated by the examination of the $\widetilde{P S F}$ only for the main lobe.

Then, in order to appreciate the validity of the approximation of the PSF still further, fig. 8 reports the behavior of the $2 \Delta \theta_{t h}$ main lobe width, defined as $\left|\widetilde{P S F}\left(\theta_{0} \mp \Delta \theta_{t h}, \theta_{0}\right)\right|=$ $0.6\left|\widetilde{P S F}\left(\theta_{0}, \theta_{0}\right)\right|$, for both the actual and the approximated PSFs. As expected, these functions exhibit a general spatially variant behavior, i.e. a dependence on the reference angle $\theta_{0}$ for each different angle geometry. Again, the results of (22) and (23) agree quite well and point out the angle dependent behavior of the functions. Accordingly, it can be expected that a general angle geometry may not be able to radiate identical focusing beams pointing at different directions.
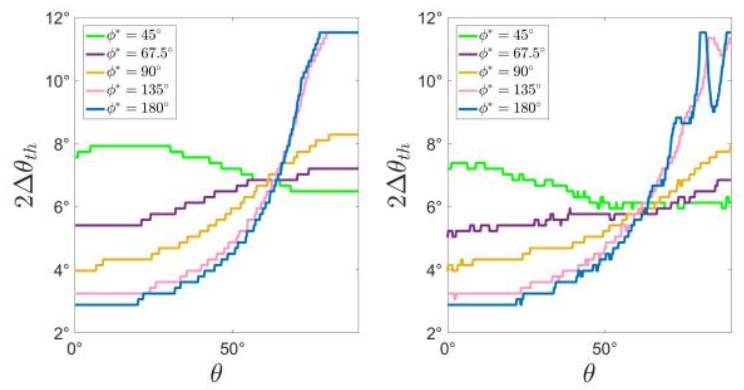

Fig. 8 Main lobe width $2 \Delta \theta_{t h}$ of the $\widetilde{P S F}$ (leftt panel) and the actual PSF (right panel) for different angle geometries .

A more general appreciation of these results can be gained by the investigation of the behavior of the $\widetilde{P S F}\left(\theta, \theta_{0}\right)$ (27) within its main lobe around the $\theta_{0}$ direction, when we assume $z_{01}=0$ for the sake of simplicity, which is performed in detail in the Appendix.

It shows that the behavior of the function main lobe scales as $b / \lambda$, in all circumstances. Accordingly, a synthetic and unified representation of the results about the main lobe Half Power
Beam Width (HPBW) $\Delta \theta$ can be gained by representing the implicit function

$\left|\widetilde{P S F}\left(t, \theta_{0}, \phi^{*}\right)\right|=1 / \sqrt{2}$

where $t=\beta b \Delta \theta$. Fig. 9 displays its contour plot for different $t$ values as a function of $\theta_{0}$ and $\phi^{*}$.

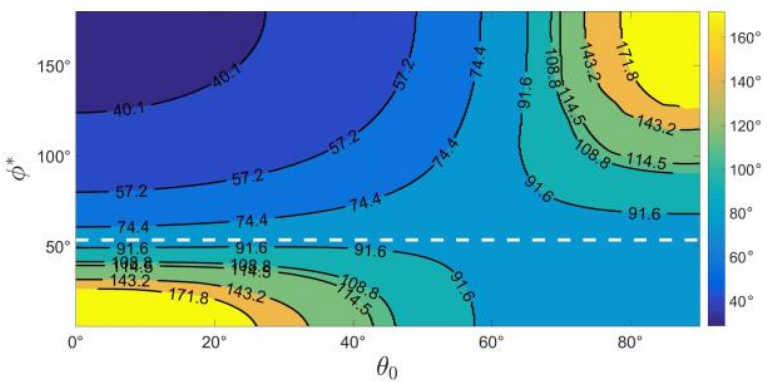

Fig. 9 Contour plot of the implicit function (28) for different $t=\beta b \Delta \theta$ values as a function of $\phi^{*}=2\left(180^{\circ}-\phi_{01}\right)$ and $\theta_{0}$.

At this point, since the main lobe widths of the beams pointing at different $\theta_{0}$ directions vary with the $\phi_{01}$ angle, we can investigate whether an optimal value of the source angle $\hat{\phi}_{01}$ exists so that the beams have nearly identical widths. To this end, we may consider the arbitrary maximum $\theta_{0}$ directions, but it is more convenient to resort to the simplest expressions of $|\widetilde{P S F}|$, that is the ones for $\theta_{0}=0^{\circ}, \pm 90^{\circ}$, and use them to estimate $\hat{\phi}_{01}$ by assuring the same $\Delta \theta_{t h}$ at these three observation directions. Therefore, by ensuring $\left|\widetilde{P S F}\left(\Delta \theta, 0^{\circ}\right)\right|=\left|\widetilde{P S F}\left( \pm 90^{\circ}+\Delta \theta, \pm 90^{\circ}\right)\right|$ for some common $\Delta \theta$ the condition

$\left|\cos \hat{\phi}_{01}\right|=2\left|\sin \hat{\phi}_{01}\right|$

is obtained, so providing, $\hat{\phi}_{01}=153.5^{\circ}$ since $90^{\circ} \leq \hat{\phi}_{01} \leq$ $180^{\circ}$. Accordingly, the corresponding angle aperture reads as

$\hat{\phi}^{*}=53.3^{\circ}$.

which is reported by a dashed line in fig. 9 .

It can be appreciated that, as well known, the linear source $\left(\phi^{*}=180^{\circ}\right)$ allows to achieve the smallest beam along the broadside direction for a fixed overall electrical length of the source, but the beam broadens at most approaching the endifire direction. On the contrary, with an optimized angle source $\left(\phi^{*}=\hat{\phi}^{*}\right.$, and $\left.t^{*}=89.4^{\circ}\right)$, identical beams can be radiated by a source with the same overall electrical length, but, as a tradeoff, their width is not much greater than double the value of a linear source.

\section{APPLICATION TO PATTERN SYNTHESIS}

Beyond their theoretical relevance in inverse problem solution, the results of the previous Sections can attract the interest of the antenna designer, too, as they may help in defining an optimal geometry for a conformal antenna composed of two linear sources. As an application, we consider 
the case of a radar surveillance application, where the antenna is required to radiate identical beams pointing at different directions within a large angular domain, so providing, possibly, a hemispherical coverage. To this end the analysis of the previous Section about the features of the PSF may help considerably in establishing a comparison between different angle geometries. In addition, the availability of approximate, but rather accurate, analytical expression for it may reduce the comparison time.

So, for instance, let us require to radiate an identical beam, whose maximum points at different $\theta_{0}$ directions within the interval $\left(0^{\circ}, 90^{\circ}\right)$, with a prefixed $-3 \mathrm{~dB}$ HPBW equal to $\widetilde{\Delta \theta}=2.5^{\circ}$. Therefore, the optimal source angle aperture to be chosen is (30). This choice leads to a source with a NDF approximately equal to (15) as shown in Section II, and numerically confirmed in Section III. The required source length is, obviously, $b=t * /(\beta \widetilde{\Delta \theta})=5 \lambda$ in the present instance. A $4 \mathrm{~b}$ linear source is able to radiate such a wide beam in the angular sector $\left|\theta_{0}\right| \leq \theta_{0 \max }$, where $\cos \theta_{0 \max }=$ $79.7^{\circ} /\left(2 t^{*}\right)$, (in this case $\theta_{0 \max }=63.4^{\circ}$ ). Therefore, the angular coverage of the radiated patterns can be expected to be extended by this choice of the angle source geometry.

In order to numerically confirm the expectations, we performed the power synthesis of the following pattern by the orthogonal projections method [23]. It consists of a main lobe corresponding to the above considered angle source, while all side lobes are constrained to be lower than $-18 \mathrm{~dB}$, i.e.

$$
\begin{array}{cc}
|U(\theta)|= \\
\left\{\begin{array}{cc}
\left|\operatorname{sinc}\left[2 \beta b \sin \hat{\phi}_{01}\left(\theta-\theta_{0}\right)\right]\right| & \text { for }\left|\theta-\theta_{0}\right|<45^{\circ} \\
0.126 & \text { elsewhere }
\end{array}\right.
\end{array}
$$

For the sake of comparison, a linear source of the same $4 b=$ $20 \lambda$ total length has been considered, too, (i.e. with the same $\mathrm{NDF}$ ) and the same radiation patterns are considered, except for the direction $\theta_{0}=90^{\circ}$. At each step of the iterative procedure, the current far field $U(\theta)$ is projected onto the subspace of the physically realizable radiation pattern by

$\widetilde{U}(\theta)=\sum_{n=1}^{N D F} c_{n} u_{n}(\theta)$

where

$c_{n}=\int_{-\pi}^{\pi} U(\theta) u_{n}^{*}(\theta) d \theta$

and the $\left\{u_{n}(\theta)\right\}$ are the left singular functions of the SVD of (7), so pertaining to the exact forward operator.

The obtained patterns are displayed in Fig. 10 and they confirm that the adoption of an angle source with the optimal choice of the $\phi^{*}$ angle assures pretty identical performances over the whole observation domain, while the linear source provides wider main lobes and increasing side lobes towards the endfire direction.
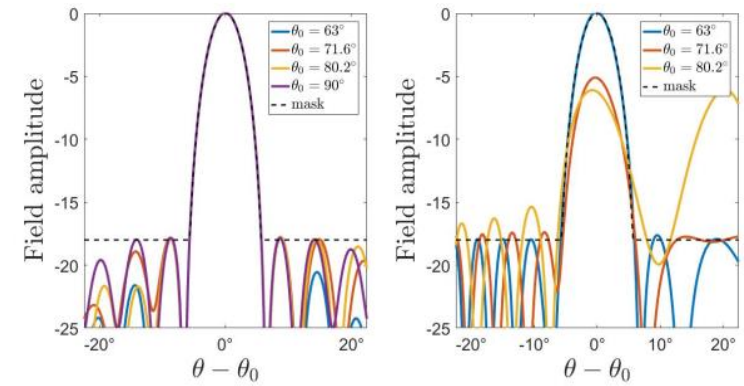

Fig. 10 Synthesized radiation pattern of identical beams pointing at different $\theta_{0}$ directions for an angle source with $\phi^{*}=\hat{\phi}^{*}$ (left panel) and a linear source (rigth panel), with the same total length $p=20 \lambda$. The assigned 'mask' is depicted under a dashed black line.

\section{LINEAR ARRAYS}

Identical discrete sources arranged to provide a conformal array antenna are of paramount interest in several applications, because of their flexibility. Therefore, it would of interest to apply the approach of the previous Sections to the practical instances of the radiation of a combination of linear arrays of identical elements. In addition, the role of the radiation pattern of the individual array element can be included in the analysis, too.

The pertinent fundamental operator, the counterpart of (1), reads now as

$E_{n}(\theta)=h\left(\theta-\theta_{n}\right) \sum_{m=-N_{a n}}^{N_{a n}} I_{n m} e^{j \beta\left[x_{n m} \sin \theta+z_{n m} \cos \theta\right]}$

where $\quad x_{n m}=x_{0 n}-m \Delta s \sin \phi_{0 n} \quad$ and $\quad z_{n m}=z_{0 n}-$ $m \Delta s \cos \phi_{0 n}$ are the rectangular coordinates of the $m-t h$ array element of the $n-t h$ line, $\Delta s$ is the element spacing, assumed equal for all linear arrays, $2 N_{a n}+1$ is the total number of the array elements of the $n-t h$ line, and $h(\cdot)$ stands for the element factor whose maximum points at a direction $\theta_{n}=\phi_{0 n}-90^{\circ}$ orthogonal to the source line. In addition, in order to account for the array element capability to radiate towards only a half-plane, we assume $h(\theta) \neq 0$, only for $|\theta|<$ $90^{\circ}$.

Equation (34) allow us to define a radiation operator $\mathcal{D}_{n}$ for each source as

$\mathcal{D}_{n}: I_{n} \in \mathcal{C}^{2 N_{a n}+1} \rightarrow E_{n} \in L_{\left[\phi_{0 n}-\pi, \phi_{0 n}\right]}^{2}$

where $L_{[,,]}^{2}$ is the set of all the finite energy functions supported in the interval of the subscript and $\underline{I}_{n}=\left\{I_{n m}\right\}$ is the vector whose components are the excitation coeffients of the $n-t h$ array.

Following the same formulation of the problem as in Section II, a total field can be defined as the superposition of the individual $E_{n}(\theta)$ fields, and a composed operator $\mathcal{D}$ defined as

$\mathcal{D}(\underline{I})=\left[\mathcal{D}_{1} \mathcal{D}_{2}\right]\left[\begin{array}{ll}\underline{I}_{1} & \underline{I}_{2}\end{array}\right]^{t}$,

for the case of a source composed of only two linear arrays, to be considered for the sake of simplicity. 
Again, it is of interest to consider the SVD of (36), and in particular, the singular values behavior in order to establish the NDF of the pertinent source. To this end, we follow the same approach as above and so introduce the adjoint operator of $\mathcal{D}_{n}$ , as

$$
\begin{gathered}
\mathcal{D}_{n}^{\dagger}(E)=\int_{-\phi_{0 n}-\pi}^{\phi_{0 n}} h^{*}\left(\theta-\theta_{n}\right) E(\theta) \\
e^{-j \beta\left[x_{n m} \sin \theta+z_{n m} \cos \theta\right]} d \theta
\end{gathered}
$$

and next, the analysis of the operator $\left(\mathcal{D}^{\dagger} \mathcal{D}\right)(\underline{I})$ leads to discuss the operator

$\left(\mathcal{D}_{i}^{\dagger} \mathcal{D}_{j}\right)\left(\underline{I}_{j}\right)=\underline{\underline{K}}_{i j} \underline{I}_{j}$

Each elements of the matrix $\underline{\underline{K}}_{i j}=\left\{K_{j m, i l}\right\}$ reads as

$$
\begin{gathered}
K_{j m, i l}=\int_{\theta} h^{*}\left(\theta-\theta_{i}\right) \\
h\left(\theta-\theta_{j}\right) e^{j \beta\left[\left(x_{j m}-x_{i l}\right) \sin \theta+\left(z_{j m}-z_{i l}\right) \cos \theta\right]} d \theta
\end{gathered}
$$

if we introduce the indices $i, j \in\{1,2\}$ to specify which source we are referring to. Two observations are now in order concerning the coefficients $K_{j m, i l}$ of (39) to evaluate their magnitude. First, they are similar to (12), as the integrand is the same except for additional slowly varying functions. Second, the integration domains $\Theta=\left[\phi_{0 i}-\pi, \phi_{0 i}\right] \cap\left[\phi_{0 j}-\pi, \phi_{0 j}\right]$ are markedly different when $i=j$ with respect to case of $i \neq j$. In fact, in the second instance, it can be considerably reduced for $\phi_{0 i}$ very different from $\phi_{0 j}$, even if the elements locations of the two arrays are close. This means that it can be expected that $\left\|\underline{\underline{K}}_{i j}\right\|_{i=j}>\left\|\underline{\boldsymbol{K}}_{i j}\right\|_{i \neq j}$. Therefore, since we are interested in evaluating $\left\|\left(\mathcal{D}_{i}^{\dagger} \mathcal{D}_{j}\right)\left(\underline{I}_{j}\right)\right\|$ via its upper bound $\left\|\left(\mathcal{D}_{i}^{\dagger} \mathcal{D}_{j}\right)\left(\underline{I}_{j}\right)\right\|<\left\|\underline{\underline{K}}_{i j}\right\|\left\|\underline{I}_{j}\right\|$, we can conlude, as in Section III, that the off-diagonal terms of $\left(\mathcal{D}^{\dagger} \mathcal{D}\right)(\underline{I})$ are negligible with respect to the diagonal ones, and again that the total NDF of the combination of the two linear arrays is the sum of the individual NDF of each array.

Accordingly, if the individual arrays do not intersect, the NDF of (35) is approximately $\left[\frac{2 \beta}{\pi}\left(2 N_{a n}+1\right) \Delta s\right]$, which is in agreement with Section II since a linear array source is a particular case of a linear continuous source of the same total electrical length.

The numerical evaluation of the singular values of (36), shown in fig 11 , for an angle array source with $\Delta s=\lambda / 2, N_{s 1}=$ $N_{s 2}=\left(2 N_{a}+1\right)=21$, element factor

$$
h(\theta)=\operatorname{rect}\left(\frac{\theta}{\pi}\right) \cos (\theta)
$$

where rect $\left(\frac{\theta}{\pi}\right)$ stands for the rectangular pulse function of $\pi$

\footnotetext{
${ }^{1}$ We recall that, for small $\alpha, D_{M}(\alpha) \simeq \sin [(2 M+1) \alpha / 2] /[(2 M+1) \alpha / 2]$, so that most analytical details obtained for a continuous source hold for an
} array, too. width, and different $\phi^{*}$, confirms the expectations. In fact, the number of significant singular values can be assumed to be 40 , that is the summation of the NDF of each source array.

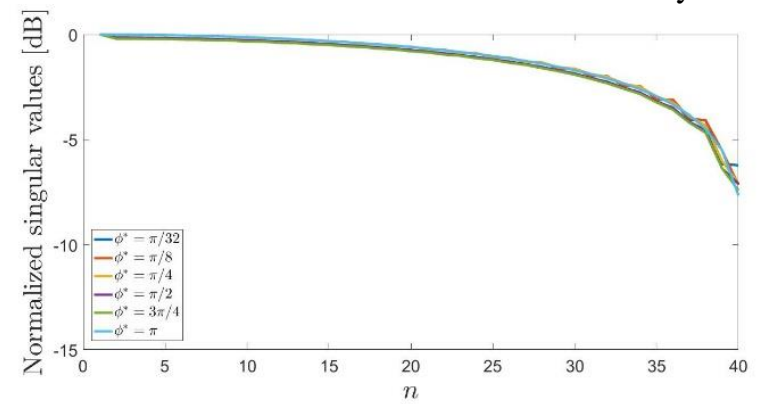

Fig. 11 Normalized singular values of (45) for angle arrays with $\Delta s=\lambda / 2$, $N_{S_{1}}=N_{S_{2}}=21$ and a cosine factor element (49), for different $\phi^{*}$ angle.

Also for a discrete source an approximated PSF function as in (23) of Section IV can be defined as

$\overline{P S F}\left(\theta, \theta_{0}\right)=\left(\mathcal{D D}^{\dagger}\right) \delta\left(\theta-\theta_{0}\right)$

which, since,

$$
\begin{gathered}
\left(\mathcal{D}_{n} \mathcal{D}_{n}^{\dagger}\right) \delta\left(\theta-\theta_{0}\right)=\left(2 N_{a}+1\right) h\left(\theta-\theta_{n}\right) h^{*}\left(\theta_{0}-\theta_{n}\right) \\
e^{j \beta\left[x_{0 n}\left(\sin \theta-\sin \theta_{0}\right)+z_{0 n}\left(\cos \theta-\cos \theta_{0}\right)\right]} \\
D_{N_{S_{n}}}\left\{\beta \Delta s\left[\cos \left(\theta_{0}-\phi_{0 n}\right)-\cos \left(\theta-\phi_{0 n}\right)\right]\right\}
\end{gathered} .
$$

is written in closed form by means of the so-called Dirichlet kernel $D_{M}(\alpha)=\sin [(2 M+1) \alpha / 2] /[(2 M+1) \sin (\alpha / 2)]^{1}$ as a summation of similar terms with different arguments like in (27), provided $\theta_{0} \in\left[\phi_{0 n}-180^{\circ}, \phi_{0 n}\right]$.

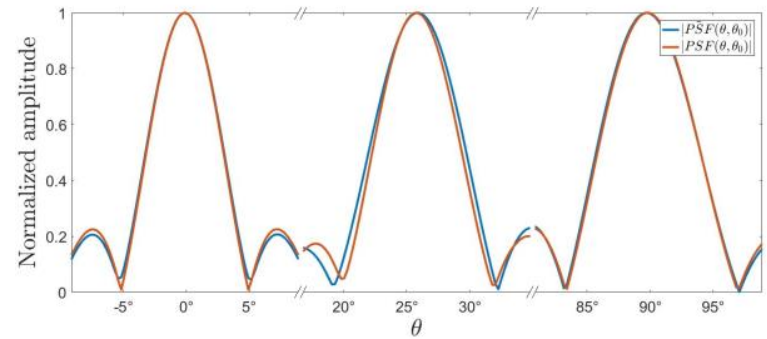

Fig. 12 Comparison of normalized actual (red line) and approximate (blue line) PSFs for an angle source array with $\phi^{*}=67.5^{\circ}$ for $\theta_{0}=0^{\circ}, 25.8^{\circ}, 90^{\circ}$; each array is composed of $N_{s}=11$ elements spaced at, $\Delta s=0.5 \lambda$.

Fig. 12, providing a comparison between the exact and the approximated PSFs, shows that an analysis similar to the one of Section IV about the behaviour of the main beam HPBW can be performed exploiting the closed form expression that can be obtained by (42).

In addition, by the same small angle reasoning, it can be recognized that the $\overline{P S F}$ mainly depends on $t=\beta \Delta s\left(2 N_{a}+\right.$ 1) $\Delta \theta / 2$, just like (28), since the slowly varying terms related to the element factor do not significantly affect the results. Therefore, again, it is worth introducing and examining the 
implicit function

$\left|\overline{P S F}\left(t, \theta_{0}, \phi^{*}\right)\right|=1 / \sqrt{2}$

which is reported under fig. 13 as a contour plot of $\mathrm{t}$ as a function of $\theta_{0}$ and $\phi^{*}$ for the case of two identical linear array arranged at an angle geometry.

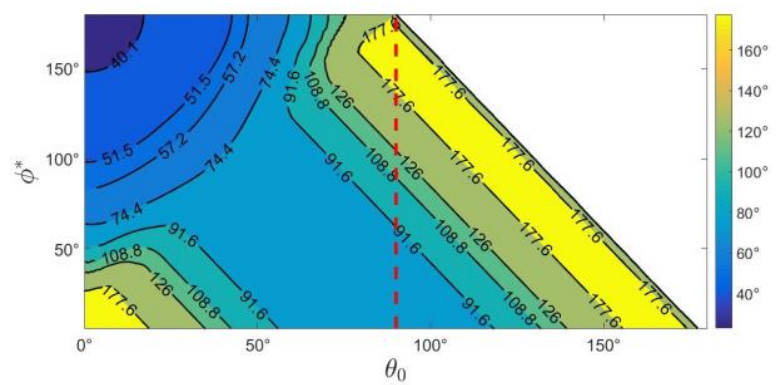

Fig. 13 Contour plot of the implicit function (43) for different $t$ values as a function of $\phi^{*}=2\left(180^{\circ}-\phi_{01}\right)$ and $\theta_{0}$. The red dashed line highlights the function $t\left(\phi^{*}, 90^{\circ}\right)$ for $\theta_{0}=90^{\circ}$. Because of the element factor (40), in the upper right triangle domain, no array radiates, in the symmetric upper left triangle both arrays radiate and in the remaining lower triangle region only one array contributes to the far field. The line $\phi^{*}=180^{\circ}$ corresponds to a single linear array lying on the $\mathrm{x}$ axis.

This plot may be helpful in revealing whether an optimal geometrical configuration of an angle array exists to radiate equal main beams pointing at different directions within an angular domain larger than the one of a single linear array.

In fact, as an example, let us consider an application where it is required to radiate identical focusing beams with $\Delta \theta_{t h}^{*}=2.5^{\circ}$ HPWB by arrangements of linear arrays whose elements are $\Delta s=\lambda / 2$ spaced away with the factor element dictated by (40). A single 41 element linear array satisfies this requirement for $\left|\theta_{0}\right|<\theta_{0 \max }=60^{\circ}$, but in order to increase $\theta_{0 \max }$ to $90^{\circ}$ an angle configuration of two identical 21 element linear arrays may be attractive. To this end, first we evaluate $t\left(\Delta \theta_{t h}^{*}\right)=$ $81.4^{\circ}$ as a synthetic performance index accounting for the required $\mathrm{HPBW}$ and next we investigate, by means of the results of fig. 13 , whether a $\phi^{*}=\hat{\phi}^{*}$ value does exist such that $t\left(\hat{\phi}^{*}, \theta_{0}\right) \leq 81.4^{\circ}, \forall \theta_{0} \leq 90^{\circ}$. Fig. 14, plotting both $\max _{\theta_{0}} t\left(\phi^{*}, \theta_{0}\right)$ and $t\left(\phi^{*}, 90^{\circ}\right)$ functions, is helpful since it allows to establish, for each $\phi^{*}$, whether the corresponding angle geometry is able to radiate beams whose largest HPBW is at most the assigned $\Delta \theta_{t h}^{*}$. Since this is not the case, as the minimum $t_{\max }\left(\hat{\phi}^{*}\right)=92.2^{\circ}$, we can conclude that, in order to reach this number, the array configuration should be changed and the number of elements should be increased by the $92.2^{\circ} / 81.4^{\circ}$ factor, i.e. to 25 . On the contrary, if this were not viable for some reason, identical beams would be radiated as well, but their HPBWs should be increased by $13 \%$. In addition, the optimal source angle geometry value $\hat{\phi}^{*}=63^{\circ}$ can be read under fig. 14, too. Accordingly, an incremental increase in the number of radiators can allow to achieve a wider angular coverage by properly combining the simultaneous radiation of two linear arrays.

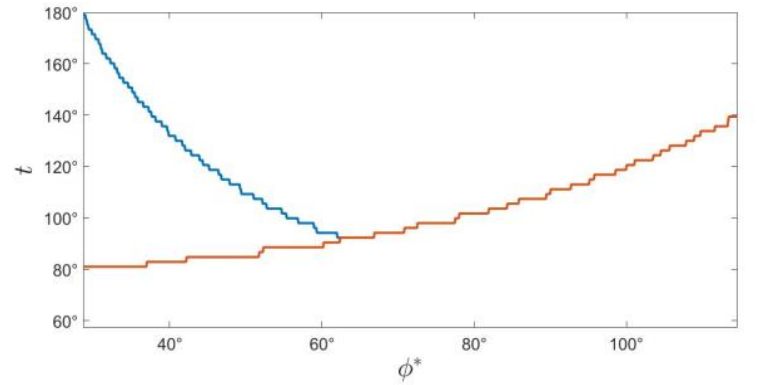

Fig. 14 Behavior of the $\max _{\theta_{0}} t\left(\phi^{*}, \theta_{0}\right)$ (blue line) and $t\left(\phi^{*}, 90^{\circ}\right)$ (red line) functions as extracted from fig. 13 .

Fig. 15 confirms the expectations as displays the results of the synthesis of the same pattern as (31) by the projection method, for the above discussed angle array

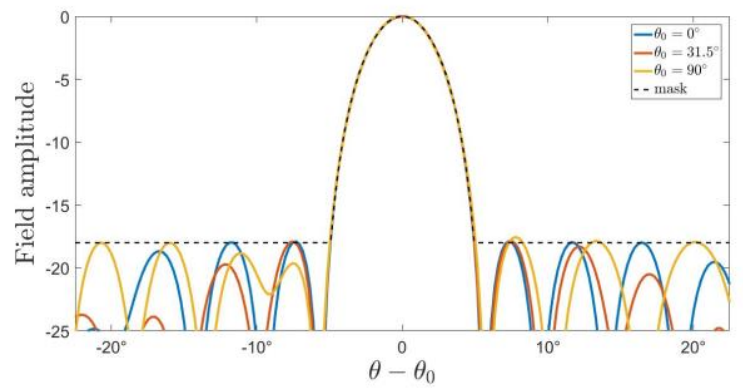

Fig. 15 Synthesized radiation pattern of identical beams pointing at different $\theta_{0}$ directions for an angle source with $\phi^{*}=\widehat{\phi}^{*}$ and $N_{a}=\widehat{N}_{a}$. The assigned 'mask' is depicted under a dashed black line.

\section{CONCLUSIONS}

In this work we extended the NDF results of a single line of [14] to a collection of linear sources. Following an inverse problem approach, we highlighted the role of the distances between the lines by resorting to an approximate evaluation of the norm of an auxiliary operator. It turns out that, when the lines are not too close and do not intersect, the total NDF is the sum of the NDF of all individual lines. Thus, for a closed linear source, i.e. a polygonal one, the perimeter determines the NDF, as for a circumference.

Next, we investigated the class of radiation patterns that can be radiated by the lines, focusing on the comparison between a single linear source and a combination of two of them to provide an angle source. An approximate, closed form, evaluation of the relevant PSF, which was properly compared with the exact one as far as its main lobe is concerned, led us to the determination of an optimal angle geometry allowing to radiate identical beams within an angular sector larger than the ones of a single line.

Finally, we applied the whole approach to the synthesis of a collection of two linear arrays forming an angle, a basic geometry in multi-faceted array for radar surveillance applications, which allows to predict their capabilities of achieving semicircular scanning with identical beams focusing at different directions. 


\section{APPENDIX}

Hereafter we discuss the details of the properties of the $\widetilde{P S F}$ around the main beam direction.

First of all, it can be easily appreciated that for a $4 b$ long single linear vertical source

$\widetilde{P S F}\left(\theta, \theta_{0}\right)=4 b \operatorname{sinc}\left\{2 \beta b\left(\sin \theta-\sin \theta_{0}\right)\right\}$,

which for $\theta \cong \theta_{0}+\Delta \theta$ reads as

$\widetilde{P S F}\left(\theta, \theta_{0}\right) \simeq 4 b \operatorname{sinc}\left\{2 \beta b \Delta \theta \cos \theta_{0}\right\}$

and that, for $\phi_{01}$ not too close to $180^{\circ}$ and small $\theta$,

$\widetilde{P S F}(\Delta \theta, 0) \simeq 4 b \operatorname{sinc}\left\{2 \beta b \Delta \theta \sin \phi_{01}\right\}$,

as $x_{01}=b \sin \phi_{01}$. This means that an angle source radiates around the direction $\theta_{0}=0^{\circ}$ like a $\sin \phi_{01}$ times shorter linear source of the same total $4 b$ length.

Next, we rewrite (27) by the sum-to-product trigonometric identities so that

$$
\begin{gathered}
\widetilde{P S F}\left(\theta_{0}+\Delta \theta, \theta_{0}\right)=2 b\left\{e^{j 2 \beta x_{01} \cos \left(\theta_{0}+\frac{\Delta \theta}{2}\right) \sin \frac{\Delta \theta}{2}}\right. \\
\operatorname{sinc}\left[\beta b 2 \sin \left(\theta_{0}-\phi_{01}+\frac{\Delta \theta}{2}\right) \sin \frac{\Delta \theta}{2}\right]+ \\
e^{\left.-j 2 \beta x_{01} \cos \left(\theta_{0}+\frac{\Delta \theta}{2}\right) \sin \frac{\Delta \theta}{2} \operatorname{sinc}\left[\beta b 2 \sin \left(\theta_{0}+\phi_{01}+\frac{\Delta \theta}{2}\right) \sin \frac{\Delta \theta}{2}\right]\right\}} .
\end{gathered}
$$

Then, we expand the trigonometric functions under the Taylor series in (A4) computed around the $\theta_{0}$ for non null $0-$ th order term, that is for $\theta_{0} \neq \pm 90^{\circ}, \pm \phi_{01}, \pm\left(180^{\circ}-\phi_{01}\right)$, so that

$$
\begin{gathered}
\widetilde{P S F}\left(\theta_{0}+\Delta \theta \theta_{0}\right) \simeq 2 b\left\{e^{j \beta b \Delta \theta \sin \phi_{01} \cos \theta_{0}}\right. \\
\operatorname{sinc}\left[\beta b \Delta \theta \sin \left(\theta_{0}-\phi_{01}\right)\right]+ \\
e^{\left.-j \beta b \Delta \theta \sin \phi_{01} \cos \theta_{0} \operatorname{sinc}\left[\beta b \Delta \theta \sin \left(\theta_{0}+\phi_{01}\right)\right]\right\}}
\end{gathered} .
$$

This shows that, for every $\theta_{0}$ direction, the $\widetilde{P S F}$ scales as $b / \lambda$ around the main lobe, although its width can be different.

Since the adopted Taylor expansion is not valid everywhere, let us investigate what happens to the PSF main lobe when $\theta_{0}=$ $\left.\pm 90^{\circ}, \pm \phi_{01}, \pm\left(180^{\circ}-\phi_{01}\right)\right)$.

For $\theta_{0}= \pm 90^{\circ}$, (A4) becomes

$$
\begin{gathered}
\widetilde{P S F}\left(\theta_{0}+\Delta \theta, \pm 90^{\circ}\right)=2 b\left\{e^{\mp j 2 \beta b \sin \phi_{01}\left(\sin \frac{\Delta \theta}{2}\right)^{2}}\right. \\
\operatorname{sinc}\left[\beta b 2 \cos \left(\phi_{01}-\frac{\Delta \theta}{2}\right) \sin \frac{\Delta \theta}{2}\right]+ \\
\left.e^{ \pm j 2 \beta b \sin \phi_{01}\left(\sin \frac{\Delta \theta}{2}\right)^{2}} \operatorname{sinc}\left[\beta b 2 \cos \left(\phi_{01}+\frac{\Delta \theta}{2}\right) \sin \frac{\Delta \theta}{2}\right]\right\}
\end{gathered}
$$

which, in virtue of the small $\Delta \theta$ angle approximation, becomes

$$
\begin{gathered}
\widetilde{P S F}\left(\theta_{0}+\Delta \theta, \pm 90^{\circ}\right) \simeq 4 b \\
\cos \left(\beta b \Delta \theta^{2} \sin \phi_{01} / 2\right) \operatorname{sinc}\left[\beta b \Delta \theta \cos \phi_{01}\right]
\end{gathered}
$$

where the cosine function can be approximated by one for small arguments.

When $\theta_{0}= \pm \phi_{01}$, (A4) becomes

$$
\begin{gathered}
\widetilde{P S F}\left(\theta_{0}+\Delta \theta, \pm \phi_{01}\right)=2 b\left\{e^{\mp j 2 \beta x_{01} \cos \left(\phi_{01} \pm \frac{\Delta \theta}{2}\right) \sin \frac{\Delta \theta}{2}}\right. \\
\operatorname{sinc}\left[\beta b 2 \sin \left(2 \phi_{01} \pm \frac{\Delta \theta}{2}\right) \sin \frac{\Delta \theta}{2}\right]+ \\
\left.e^{ \pm j 2 \beta x_{01} \cos \left(\phi_{01} \pm \frac{\Delta \theta}{2}\right) \sin \frac{\Delta \theta}{2}} \operatorname{sinc}\left[\beta b 2\left(\sin \frac{\Delta \theta}{2}\right)^{2}\right]\right\}
\end{gathered}
$$

that can be approximated, if $\Delta \theta$ is sufficiently small, as

$\widetilde{P S F}\left(\theta_{0}+\Delta \theta, \pm \phi_{01}\right) \simeq 2 b e^{\mp j \beta b \Delta \theta \cos \phi_{01} \sin \phi_{01}}$
$\left\{e^{\mp j \beta b \Delta \theta \cos \phi_{01} \sin \phi_{01}} \operatorname{sinc}\left[\beta b \Delta \theta \sin \left(2 \phi_{01}\right)\right]+\right.$
$\left.e^{ \pm j \beta b \Delta \theta \cos \phi_{01} \sin \phi_{01}} \operatorname{sinc}\left[\beta b \Delta \theta^{2} / 2\right]\right\}$

Since $\Delta \theta^{2}$ is very small, we can further assume that the second sinc function is 1 , obtaining

$$
\begin{gathered}
\widetilde{P S F}\left(\theta_{0}+\Delta \theta, \pm \phi_{01}\right)=2 b\left\{e^{\mp j \beta b \Delta \theta \cos \phi_{01} \sin \phi_{01}}\right. \\
\operatorname{sinc}\left[\beta b \Delta \theta \sin \left(2 \phi_{01}\right)\right]+ \\
\left.e^{ \pm j \beta b \Delta \theta \cos \phi_{01} \sin \phi_{01}}\right\}
\end{gathered}
$$

The last case to be considered is for $\theta_{0}= \pm\left(180^{\circ}-\phi_{01}\right)$, leading (A4) to become

$$
\begin{gathered}
\widetilde{P S F}\left(\theta_{0}+\Delta \theta, \pm\left(180^{\circ}-\phi_{01}\right)\right)= \\
2 b\left\{e^{\mp j 2 \beta x_{01} \cos \left(\phi_{01} \mp \frac{\Delta \theta}{2}\right) \sin \frac{\Delta \theta}{2}} \operatorname{sinc}\left[\beta b 2 \sin \left(2 \phi_{01} \mp \frac{\Delta \theta}{2}\right) \sin \frac{\Delta \theta}{2}\right]+\right. \\
\left.e^{ \pm j 2 \beta x_{01} \cos \left(\phi_{01} \mp \frac{\Delta \theta}{2}\right) \sin \frac{\Delta \theta}{2}} \operatorname{sinc}\left[\beta b 2\left(\sin \frac{\Delta \theta}{2}\right)^{2}\right]\right\}
\end{gathered}
$$

that, for small $\Delta \theta$, returns

$$
\begin{aligned}
& \widetilde{P S F}\left(\theta_{0}+\Delta \theta, \pm\left(\pi-\phi_{01}\right)\right) \simeq 2 b \\
& \left\{e^{\mp j \beta b \Delta \theta \sin \phi_{01} \cos \phi_{01}} \operatorname{sinc}\left[\beta b \Delta \theta \sin \left(2 \phi_{01}\right)\right]+\right. \\
& \left.e^{ \pm j \beta b \Delta \theta \sin \phi_{01} \cos \phi_{01}} \operatorname{sinc}\left[\beta b \Delta \theta^{2} / 2\right]\right\}
\end{aligned}
$$

which, by assuming the second sinc equal to one, finally gives

$$
\begin{gathered}
\widetilde{P S F}\left(\theta_{0}+\Delta \theta, \pm\left(\pi-\phi_{01}\right)\right) \simeq 2 b\left\{e^{\mp j \beta b \Delta \theta \sin \phi_{01} \cos \phi_{01}}\right. \\
\quad \operatorname{sinc}\left[\beta b \Delta \theta \sin \left(2 \phi_{01}\right)\right]+e^{\left. \pm j \beta b \Delta \theta \sin \phi_{01} \cos \phi_{01}\right\} .} .
\end{gathered}
$$

We can also conclude, then, that

$$
\begin{aligned}
& \widetilde{P S F}\left(\theta_{0}+\Delta \theta, \pm \phi_{01}\right)=\widetilde{P S F}\left(\theta_{0}+\Delta \theta, \pm\left(180^{\circ}-\phi_{01}\right)\right)= \\
& 2 b\left\{e^{\mp j \beta b \Delta \theta \sin \phi_{01} \cos \phi_{01} \operatorname{sinc}\left[\beta b \Delta \theta \sin \left(2 \phi_{01}\right)\right]+.} .\right. \\
& \left.e^{ \pm j \beta b \Delta \theta \sin \phi_{01} \cos \phi_{01}}\right\}
\end{aligned}
$$

\section{REFERENCES}

[1] G. H. Knittel, "Choosing the Number of Faces of a Phased-Array Antenna for Hemispherical Scan Coverage," IEEE Trans. Ant. Propagat., Vol. 13, N. 6, pp. 878-882, Nov. 1965.

[2] J.L. Kmentzo, "An Analytical Approach to the Coverage of a Hemisphere by N Planar Phased Arrays," IEEE Trans. Ant. Propagat., Vol. 15, N. 3, pp. 367-371, May 1967. 
[3] G.V. Trunk and D.P. Patel, "Optimal number of phased array faces and signal processors for horizon surveillance", IEEE Trans. Aerospace and Electronic Systems, Vol. 33, N. 3, pp. 1002-1006, 1997.

[4] I. Khalifa and R. G. Vaughan, "Geometric Design and Comparison of Multifaceted Antenna Arrays for Hemispherical Coverage", IEEE Trans. Ant. Propagat, vol. 57, no. 9, pp. 2608-2614, Sept. 2009.

[5] L. Josefsson, and P. Persson, Conformal Array Antenna Theory and Design, IEEE Press

[6] A. N. Tikhonov, and V. Y. Arsenin, Solution to ill-posed problems, Winston, 1977

[7] G. Toraldo di Francia, "Degrees of freedom of an image," J. Opt. Soc. Am., Vol. 59, N. 7, pp. 799-804, 1969.

[8] R. Piestun, and D. A. B. Miller, "Electromagnetic degrees of freedom of an optical system," J. Opt. Soc. Am. A, Vol. 17, N. 5, pp. 892-902, 2000.

[9] M. A. Maisto, R. Solimene, and R. Pierri, "Source's symmetries and priors: the effect on information content of radiated field", 2018 IEEE Int. Symp. on Antennas and Propagation, 2018.

[10] R. Solimene, M. A. Maisto, and R. Pierri, "Information Content in Inverse Source with Symmetry and Support Priors", Progress In Electromagnetics Research C, Vol. 80, pp. 39-54, 2018.

[11] Newsam, and R. Barakat, "Essential dimension as a well-defined number of degrees of freedom of finite-convolution operators appearing in optics," J. Opt. Soc. Am. A, Vol. 2, N. 11, pp. 2040-2045, 1985.

[12] G. Leone, M. A. Maisto, and R. Pierri, "Application of inverse source reconstruction to conformal antennas synthesis", IEEE Trans. Ant. Propagat, Vol. 66, N. 3, pp. 1436-1445, 2018.

[13] G. Leone, F. Munno and R. Pierri, "Fourier analysis for the reconstruction of radiating sources on conic curves", 41 th Photonics \& Electromagnetics Research Symposium (PIERS 2019), Rome, Italy, June 2019.

[14] R. Pierri, A. Liseno, F. Soldovieri, and R. Solimene, "In-depth resolution for a strip source in the Fresnel zone," J. Opt. Soc. Am. A, vol. 18, no. 2, pp. 352-359, 2001.

[15] R. Solimene, M.A. Maisto, and R. Pierri, "Sampling approach for singular system computation of a radiation operator", Journal of the Optical Society of America A, Vol 36, N. 3, pp. 353-361, 2019.

[16] G. Leone, "Source geometry optimization for hemispherical radiation pattern coverage," IEEE Trans. Antennas Propag., vol. 64, n. 5, pp .20332038, May 2016.

[17] M. Bertero, and P. Boccacci, Introduction to inverse problems in imaging, CRC press, 1998.

[18] G. Leone, F. Munno and R. Pierri, "Comparison of Some Geometries in the Inverse Source Problem", 2019 International Conference on Electromagnetics in Advanced Applications (ICEAA), Granada, Spain, Sept. 2019.

[19] D. Slepian, H.O. Pollack, "Prolate spheroidal wave functions, Fourier analysis, and uncertainty-I", Bell Syst. Techn. J., vol. 40 pp. 43-63, 1961

[20] H.J. Landau, H.O. Pollack, "The eigenvalue distribution of time and frequency limiting", J. Math. Phys., vol 77, pp. 469-481, 1980.

[21] G. Leone, M.A. Maisto, R. Pierri, "Inverse Source of Circumference Geometries: SVD Investigation based on Fourier Analysis", Progress In Electromagnetics Research M, Vol. 76, pp. 217-230, 2018.

[22] J. B. Conway, "A course in functional analysis", vol. 96, Springer Science \& Business Media, 2013.

[23] D.C. Youla, H. Webb, "Image Restoration by the Method of Convex Projections: Part 1 Theory ", IEEE Trans. Med. Imaging, Vol. 1, N. 2, pp 81-94, Oct. 1982. 